\title{
Improved Privacy Protecting in Distributed Grid Data Resource using Multiplicative Perturbation Based on Frequent Decision Classifier
}

\author{
Praveen Kumar.G, S K Mohan Rao, B.V.Swathi
}

\begin{abstract}
The enormous amount of data process in distributed grid resource holds sensitive information on centralized access. The common privacy standard needs advancement to protect the sensitive data in various sectors like sharing, legal privacy and policies to access the data. The privacy standards depend the Distributed Data Mining (DDM) approaches like Association Rule Mining algorithms (ARM), clustering, and classification methods to preserve the data from unauthorized access. But the security standards have lacked privacy-preserving rules due to high dimensionality problems of data access leads more time complexity. To overcome the problem, to propose a Multiplicative Perturbation Swapping method based on Frequent Decision Classifier (MPS-FDC). This method is adaptive to data publishing secrecy to hold the privacy standards better than association rule prediction. This optimization resolves the forecasting leakages based on Persuasive Privacy Preserving Data Mining ( $P^{2}$ PDM) to secure the data. Which this technique initially does the sanitization to reduce the dimensionality to remove un-variant the outliers. The data perturbation keeps the original data to modify using supportive noise delimiters with state matrix distortion (SMD). So the original data keep safe without effect from the outliers. The frequent rule prediction decides to classify the recurrentdata from unauthorized access to disclosing crypto- privacy policy. The proposed system improves the privacy standard compared to the ARM specification rules.
\end{abstract}

Key terms: privacy-preserving, data mining, perturbation, swapping, forecasting, sanitization, decision classifiers.

\section{INTRODUCTION}

Due to the latest rapid data security development, the privacy-preserving distributed machine learning is increasing. This paper focuses on a class of related privacy preservation reduction by resolving machine learning problems and creates two modes to provide different privacy for learning methods distributed within the network. Initially expand the learning process using the multiplicativeadditive vector method, proposed double variable perturbation and optimal variable perturbation methods to provide dynamic difference privacy based on decision classifier.

Data collection and analysis are continuously increasing due to the widespread use of more users in web resources. The accusation rule mining algorithms defend the privacy problems. Analysis of such privacy information promotes businesses and benefits the community in various fields.

\footnotetext{
Revised Manuscript Received on September 10, 2019.

Mr.Praveen Kumar.G, Research Scholar, JNTUH \& Asst.Professor, CMR Engineering College, Hyderabad, Telangana, India

Dr.S K Mohan Rao, Professor \& Principal, Gandhi Institute for Technology, Bhubaneswar,Odisha, India

Dr.B.V.Swathi, Professor , Geethanjali College of Engineering \& Technology,Hyderabad, Telangana, India
}

However, the flow of this storage and important data provides serious privacy concerns. When protecting privacy, methods that allow the data to be extracted from the data are known for Privacy-Preserving Data Mining (PPDM) techniques. This examines the most relevant PPDM techniques from Metrics used to evaluate frequent relational accessing storage records and measurement to provide regular applications of PPDM systems in Apriority-based Elephant Herd Optimization (AEHO) and related fields. Furthermore, PPDM's current challenges and open issues are discussed in the literature review.

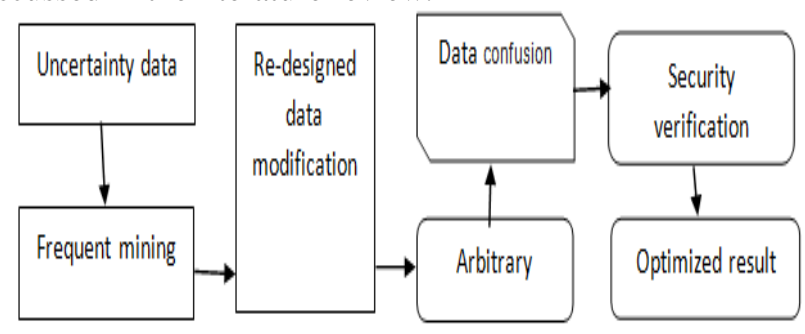

Figure 1: procedure of privacy-preserving data mining in the dispersed location

Some advantages of communication technologies are collection and analysis (sometimes critical) only possible through data. However, this can lead to unwanted privacy violations. Figure 1shows the process of privacy-preserving data mining in the distributed environment. To protect from the information leak, the privacy protection methods are created to protect the exposure of the owner whether the data is modified or not. By changing the original data However, changing the data can reduce its use, resulting in an incorrect or inaccurate extraction of knowledge through data mining.

The frequents are analyzed through the rule set, classification and clustering of the Association rule are the differential machine learning techniques, overseeing the first two learning techniques, and the latter being independent learning. The privacy-preserving among the distributed storage records produce descriptive or predictable models from data. Explanatory samples attempt to convert data into humanly explanatory explanations, while predictive models are used to predict unknown future data. Samples are made using machine learning techniques that are classified as supervised and supervised. 
Data collection and analysis are continuously increasing due to the widespread use of computer devices. Analysis of this information is to encourage businesses and benefit the community in various fields. However, the flow of this storage and key data provides serious privacy concerns. When protecting privacy, methods that allow you to extract knowledge from the data are aware of PPDM techniques. This article examines the most relevant PPDM techniques, such as the literature and metrics used to evaluate literature and measurement to provide regular applications of PPDM systems in literature and related fields. Furthermore, the current challenges and open problems of PPDM are discussed

\section{LITERATURE REVIEWS}

The privacy depends on the knowledge process based on accessing data because of various anomalies occurred in a different situation. The following are the various reviews described by different authors.

Many data mining techniques depend on the privacy standard rules such as key verification, auditing, multiplicative principles, randomization to preserve the data in distributed data publishing [1]. Most of PPDM techs use the standardization association rule mining techniques specifying principle optimization techniques [2], but the privacy doesn't depend on the data to follow the different policy. Also, the data quality changes during additional factor need to change the originality of data to protect the data [3]. To maintain the originality of data, the cryptographic techniques use the generic constructions to compute the data confidentiality to preserve the data from anomalies [4]. The randomization differentiates the data and privacy principles based on the features of data activity. Such the activities are sanitized by accessing the features of originality of data to protect [5]. By the confidence level of apriority, the various data have specific features to use the sanitization process. But circumstances to fix the margin of privacy rules in failed to access the data publishing [6]. This occurs due to leakage of originality or the modification carried out by the anomalies. The data dependencies problems are deal with multilevel trust measures to prevent the data from attackers [7]. The multilevel authentication is possessed the knowledge hiding principles to verify the privacy data or sensitive data analysis [8]. By this fact, more computation requires indirect verification leads to time complexity and data reduction [9]. The balancing factor to the complex nature in data publishing requires statistical disclosure techniques [10]. The privacy-preserving needs additional verification because of emerging data access.

The frequent access to collect information from big data analysis discloses the related topic search models using prior algorithm [11]. The forecasting measures confidence states of various relational analysis among the data. This behavioral approach doesn't reduce the dimensionality of data access [12], but not concentrating the anomalies which they have the perspective to access the sensitive information [13]. The classification of originality states is not acquired to the correct measures. At that time outsourcing data privacy rules the data are accessible through pattern relation transaction data [14]. The right data owner knows the true pattern have higher confidential value to access the data.
The context-aware techniques use the privacy rule to the decision principles make privacy data authentication [15]. To address the battle of the approach using the clustering approach to deals the summarization context-aware problem.

In big data issues, the privacy circumstances are root to swarm intelligence concepts make to the privacy perturbations [16], the most additive techniques have the perspective techniques like Particle Swarm Optimization (PSO). Carrying patient data, the fitness doesn't hold the marginal numerical values form a complex additive process [17]. The computational process optimization techniques in classificationdepend on the categorization with huge rules. The service providers have the security concerns to access the preliminary verification problems [18]. The smart grid services use the association rule mining techniques to utilize the standard behavior analysis of each user and provide the privacy [19]. The dynamic approach helms spatiotemporal issues have the relational pattern anomalies that aggregate he infinite streams of data publishing.

\subsection{Problem definition}

When a problem with perturbation trees, the boundaries between the data collection among the frequent data's are virtually uneven-parallel, the data points can be created as separate groups. Elephant heard optimization (EHO) doesn't solve this problem. The relational frequent is to construct a decision rule set of large group classification trees using a ranking function within each volume, then calculate the little value. So the privacy loses to take verification. This classification returns the duplicate, and another similar hybrid depends methods require further inspection of performance. Another problematic issue takes place the hybrid representation construct max score relevance, i.e., each time the perturbation tree is added to each leaf to compensate for the negative functions of the variables using the average of the leaf be considered as frequent access to leave the privacy.

\section{IMPLEMENTATION OF THE PROPOSED SYSTEM}

Data mining depends on knowledge learning to sensitivity analysis in Privacy-Preserving Data Mining (PPDM) protects privacy by protecting the privacy of personal data or sensitivity knowledge without sacrificing the use of data. People are well aware of the privacy of their data and are reluctant to share their important information. This leads to data mining unnecessary privacy concerns. While there are privacy controls, several methods have been proposed, but its research has now begun. To recover the problematic factor, the privacy preserving in distributed grid data resource using multiplicative perturbation based on Frequent Decision Classifier (MPS-FDC) makes the privacy policies to preserve the data. The Data processing mechanisms that protect privacy are based on its performance, data usage, uncertainty or resistance to data mining. Nevertheless, there will be no mechanism for all privacy protection, and it will 
have all kinds of capabilities to all others. On the contrary, an algorithm may be better than one on a particular scale.To propose a method that, perturbation trees, divides a set of sub-data to form similar data that uses a revision-sharing technique. The partitioned data is handled using a subprime average. Relationships between attributes are expected to be legally protected because it is shared from a combination of many covert and covert attributes. Moreover, the proposed method is computationally efficient.

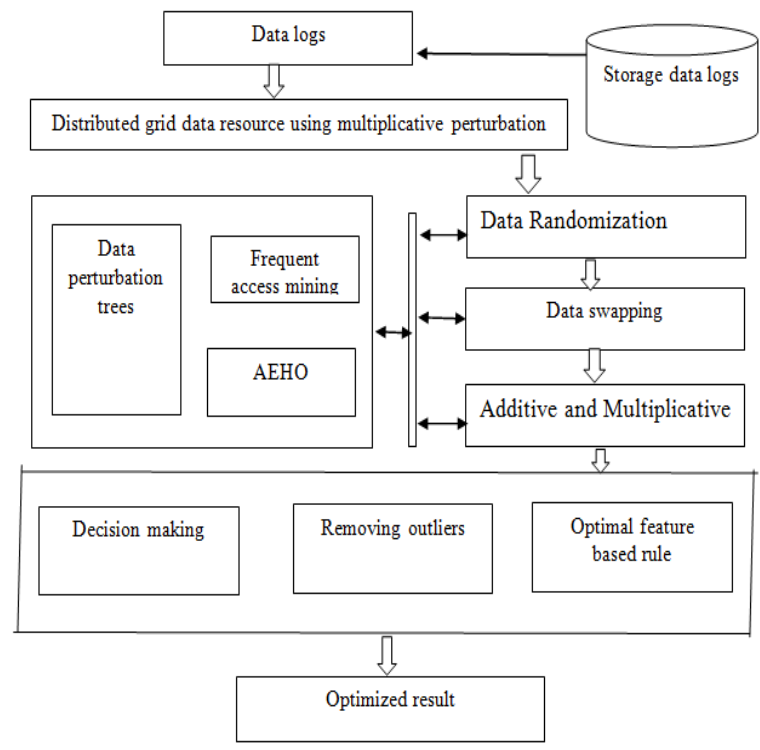

Figure 2 architecture diagrams for the proposed system

Figure 2 shows the architecture diagram for proposed system implementation. A randomized perturbation optimization algorithm is designed for finding a good perturbation with satisfactory resilience to the discussed attacks. In general, compared to randomly generated perturbations (the components $\mathrm{R}$, are randomly selected), the optimized perturbation can give significantly higher privacy guarantee. This optimization algorithm will also be used in our multiparty protocols.

One of the most widely accepted outlier techniques is remains the noise from the known distribution. In Perturbation, the original values are converted through some incorrect data values, so the numeric data calculated by data does not change counted data from the original data. Threaded data logs are not compatible with real-world recorders, so the attack can not get thoughtful connections or restore personal knowledge from the available data. By changing the data, you will be exaggerated. Additive multiplicative perturbation methods can be implemented in a centralized and distributed environment. A variation of the classical perfection technique known as randomization is a data deviation technique that includes data by modifying data values. The inconsistent response is one of the statistics. In random responses, depending on whether the data contains the correct information or the incorrect information, the central location can not be better positioned than the pre-defined entry each data is sensitive to closure of original records. If the information received from all the individual users is murmured to unauthorized users and the number of users is significantly higher, the total information of these users will be accurate whether the to optimize this implementation.

\subsection{Preliminary process}

The preliminary process takes the dataset to preprocess the high dimensionality because of duplicate records in the dataset. The dataset contains the records with noises to point differential dimensionality representation. All the records are standard to databases to process query request of information access. The transactional databases records are used to the input process which it contains frequent item sets. The frequent itemset holds relational analysiswith finite successful transactions.

Let us consider item set $\mathrm{I}=\{\mathrm{i} 1, \mathrm{i} 2,$, ,in $\}$ and finite set of items Contains the list $\mathrm{T}=\{\mathrm{t} 1, \mathrm{t} 2, \ldots \mathrm{tn}\} \rightarrow \mathrm{p}(\mathrm{i})$ items .the transaction contains $\mathrm{P}$ number items called $\mathrm{P}(\mathrm{k})$.

Definition 1. The transactional set $\mathrm{P}$ adjacent to the item set $\mathrm{I}$ in $\mathrm{P}(\mathrm{k})$ item sets.

Definition 2. Let min-sup be the minimum confidence state by the candidate for analyzing transactional data $\mathrm{P}(\mathrm{i}-\mathrm{n})$ terms, and the total transaction is $\mathrm{T}(\mathrm{n})$.

Definition 3. The support count be the minimum $\mathrm{K}$ terms of items are represented by

Min_sup_countT(K) $\rightarrow\{k$ (i) belongs to $p(i)\} /$ total terms $\mathrm{T}(\mathrm{n})$

Definition, 4.The min support count, be represented as

$$
\mathrm{Sup}_{-} \mathrm{T}(\mathrm{K})=\frac{\text { min_sup_count } t(n)}{\text { transaction }(n)}
$$

The minimum support measures to reduce the max term confidence state of each item by many transactions contain the minimum support count measure.

Minimum confidence. The minimum confidence state is analyzed using to finding the minimum item set mining item set which is reputably focused on the relation of candidate sets. This scans the datasets repeatedly to represent the relational terms using the frequent supportive measure.

\subsection{Data perturbation based on modified EHO}

The elephant population is made of several frequent sets, and the elephants in the groups live under the leadership of a root identifier. In each generation, the constant number of elephants leaves groups and goes for the selective feature which is form classification. It can be easily concluded that behavior in feature represents exploitation while leaving features are used for exploration.

\section{Algorithm}

Initialization

Set generation counter $\mathrm{t}=1$, set maximum generation MaxGen

Initialize the population and

Repeat

Sort all the elephants according to their fitness

For all clans ci in the population do

For all elephants $\mathrm{j}$ in the clan ci

Do

Update Xci,j and generate Xnew,ci,j

ifXci, $\mathrm{j}=\mathrm{X}_{\text {Optima, }}$,ci then 
Update Xci,j and generate Xnew,ci,j

End if

End for

End for

For all clans ci in the population do

Replace the worst elephant in group ci

End for

Evaluate population by the newly updated positions

Until stop criteria=false

Return the Optima solution among all population

Any EHO-distribution-based data mining algorithm operates under an implicit assumption to treat each dimension individually in the spreading approach. The relevant information for data processing protocols, such as classification, is hidden in inter-trait relationships because the understanding approach conducts different attributes individually. Thus distribution-based data mining mechanisms have an inherent advantage to the loss of hidden information protected in a variety of records

\subsection{Fitness evaluation}

Every candidate solutions in the set of all solutions $\mathrm{S}$ can be given a score, or "fitness," by a so-called fitness function. To usually write $f(s)$ to indicate the fitness of solution s. Fitness to find the $\mathrm{s}$ in $\mathrm{S}$ this has the best score.

\section{Algorithm}

Input sum of the Fitness value

For all members of the population

Sum $+=$ fitness of this individual

End for

For all members of the population

Probability $=$ sum of probabilities +

(fitness / sum)

End for

Sum of probabilities $+=$ probability

Loop until new population is full

Do this twice

Number $=$ Random between 0

and 1

For all members of a population if number > probability

but less than the next probability then you have been selected

End for

End

End loop

Create offspring

The Perturbation Approach algorithm conducts intermittent information relationships associated with the data mining processes such as sorting, which acts under an inherent assumption of treatment for each dimension of the distribution-based data processing. The privacy approach conducts different attributes individually to know the relation. Thus distribution-based data mining mechanisms have an inherent advantage to the loss of hidden information protected in a variety of records.

\subsection{Data Randomization}

The proprietary random technique is the simple to make random alignment or records in order shuffle factor and most effective approach to data mining that protects privacy.
The organization of data traditionally uses secuirty concerns through probability distributions, such as the methods of a luminous response. The technique of random system can be explained as follows. Consider a set of data indicated by $\mathrm{X}=$ $\{\mathrm{x} 1, \ldots . \mathrm{xn}\}$. The added noise components taken from FY (y) to the distinctive probability distribution for $\mathrm{Xi} \varepsilon \mathrm{X}$ edition. These noise elements are drawn freely and are represented as y1 ... yn. Therefore, a new set of corrupted $\operatorname{logs} \mathrm{x} 1+\mathrm{y} 1 \ldots \ldots \mathrm{xn}+\mathrm{yn}$. This new package posts $\mathrm{z} 1 \ldots \ldots$. $\mathrm{Zn}$. Inconsistently, some methods are numerical disorientation and aggravation of the item set. By removing the original items and adding "wrong" values to the set of properties, you can introduce many values or numerically to digital records. Some variations in randomization techniques continue to be discussed. For d-dimensional optimization problem, the position of i-th elephant group of a swarm (consisting of $\mathrm{N}$ particles, i.e., number of elephant groups) at $\mathrm{t}$-th iteration is given as $\mathrm{Xi}, \mathrm{d} \mathrm{t}=(\mathrm{xi} 1, \mathrm{xi} 2, \ldots, \mathrm{xid})$ and the velocity is represented by $\mathrm{Vi}, \mathrm{d} \mathrm{t}=(\mathrm{vi} 1, \mathrm{vi} 2, \ldots$, vid $)$. Locally best Optima solution by $\mathrm{i}$-th elephant group at current iteration is given as $\mathrm{P}(\mathrm{Optima}, \mathrm{i}, \mathrm{d}) \mathrm{t}=(\mathrm{Pi} 1, \mathrm{Pi} 2, \ldots$, Pid) and global best Optima solution is denoted by $\mathrm{G}_{\text {Optima }}, \mathrm{d}$ $\mathrm{t}=(\mathrm{G} 1, \mathrm{G} 2, \ldots, \mathrm{Gd})$. Initially, the elephant groups (position and velocity) are randomly placed throughout the search space. Each representation of records similar the velocity re[presenataion of elephants are updated according to the decision rule.

Most frequent search activities can occur at both local and global scales. In practice, adjacent optimal decision searches in the not-so-far-away neighborhood are more likely to be executed by the group than that noise far away. For this, a constant known as switching probability $\mathrm{p}$ is used to switch between global and local optimal decision searches. It is assumed that if the value of a random variable is greater than $\mathrm{p}$, common optimal decision search will be performed, else intense local optimal decision search will be executed. This randomized condition helps to reduce the probability of sticking at local optima. Global and local best search (optimal $\rightarrow$ search) solutions are updated after each iteration. As iteration proceeds, the velocities of the particles are updated in different ways for global and local search according to the following equations depending on the value of parameter $\mathrm{p}$ :

$$
\begin{aligned}
& V_{i, d}^{t+1}=V_{i, d}^{t+1} * w^{t}+\operatorname{rand}(i, d) *\left(G_{\text {Optima } i, d}^{t}-\right. \\
& \left.X_{i, d}^{t}\right) \ldots \ldots .
\end{aligned}
$$

if rand $>\mathrm{p}$ for specified iterated value

The above equation perform the local behind data perturbation to modify the $X \rightarrow p$ probability to modify the data behind at each iteration the randomized value is sector into the dot-matrix table

$$
V_{i, d}^{t+1}=V_{i, d}^{t+1} * w^{t}+\operatorname{rand}(i, d) *\left(P_{\text {Optima } i, d}^{t}-\right.
$$
$\left.X_{i, d}^{t}\right) \ldots \ldots \ldots$

if rand $<=\mathrm{p}$ for specified iterated value

The above equation perform the optimal modification based on dot matrix value whether the data perturbation to modify the $\mathrm{P} \rightarrow \mathrm{X}$ probability to modify the data behind at the each iteration 
Where rand $(1, \mathrm{~d})$ generates a d-dimensional array of random values within $[0,1]$ to denotes element-wise multiplication; $\mathrm{W}^{\mathrm{t}}$ is the inertia weight at current iteration to balance between exploration and exploitation. Then, the position of an elephant group is modified according to the following equation.

$$
X_{i, d}^{t+1}=V_{i, d}^{t+1}+X_{i, d}^{t} \cdots \cdots \cdots
$$

Where $\mathrm{T} \rightarrow \max , \mathrm{X} \rightarrow \max$, and $\mathrm{X} \rightarrow$ min represent the boundary limit of random perturbation limits represent each limit of data modification points by updating the elephant decisions group by modification value.

\subsection{Data Swapping}

A related method is data transference, where values are transmitted across different records to complete privacy protection. One of these techniques is that the total number of queues under the data is completely protected and they are not distorted. This time, it is noteworthy that other records do not follow the general doctrine of randomization that allows for the value of a freely transferred record. The table given below shows the transaction terms and redundant utility terms support by the confidence measure

\section{Table 1 transactional terms}

\begin{tabular}{|l|l|}
\hline Transaction T & $\begin{array}{l}\text { Subsequent } \\
\text { tems }\end{array}$ \\
\hline $\mathrm{t} 1$ & $\{\mathrm{~h}, \mathrm{~b}, \mathrm{y}\}$ \\
\hline $\mathrm{t} 2$ & $\{\mathrm{~h}, \mathrm{y}\}$ \\
\hline $\mathrm{t} 3$ & $\{\mathrm{~h}, \mathrm{~d}\}$ \\
\hline $\mathrm{t} 4$ & $\{\mathrm{~b}, \mathrm{e}, \mathrm{v}\}$ \\
\hline
\end{tabular}

\begin{tabular}{|l|l|}
\hline Sequence term & Support confidence \\
\hline h & 3 \\
\hline b & 2 \\
\hline y & 2 \\
\hline d & 1 \\
\hline e & 1 \\
\hline v & 1 \\
\hline
\end{tabular}

The generate minimum support confidence using $\mathrm{Ms}=$ $\sum_{i}^{n} \frac{\text { average number of dataset } / 2}{\text { total number of data }} *$ total number of transaction......

by the term transactional point of term $\mathrm{T}=\{\mathrm{T} 1, \mathrm{~T} 2, \mathrm{~T} 3$, T4\} represent as subsequent term minimum ( $\mathrm{min}$ ) value 50 $\%$ term with four transactions in average case is 2 supportive with maximal value 3 in between 2 as minimum support. The redundant term of candidate transaction is

\section{Table 3 redundant term Max:Virin Value Table 4Redundantconifidencelevel}

\begin{tabular}{|l|l|l|l|}
\hline Sequence tem & Confidence value & Sequence tem & $\begin{array}{l}\text { Redundant } \\
\text { confidence }\end{array}$ \\
\hline H & 3 & $\{H, B\}$ & 1 \\
\hline$B$ & 2 & $\{B, Y\}$ & 1 \\
\hline$Y$ & 2 & $\{H, Y\}$ & 2 \\
\hline
\end{tabular}

To generate the total confidence between min- max term supportive combination of subsequence term candidate as Cs.
$\mathrm{Cs}=$

$\sum_{i}^{n} \frac{\text { account of frequent term } / 2}{\text { candidate transaction count }} *$

total number of transaction......

By specific candidate reduction, the dimensionality is reduced by analysis the term of subsequence term from medical dataset organized by evaluation of time complexity approach.

The frequent selection is resulting from the dimensionality based on the subset sequence redundancy confidence state. By selecting a resultant class of item sets, min-max identifies the sequence by the term of a root. The frequent classes are varied obtaining new classes by interaction term data followed by root $\max (\mathrm{n})$ support followed by lead min (n) support
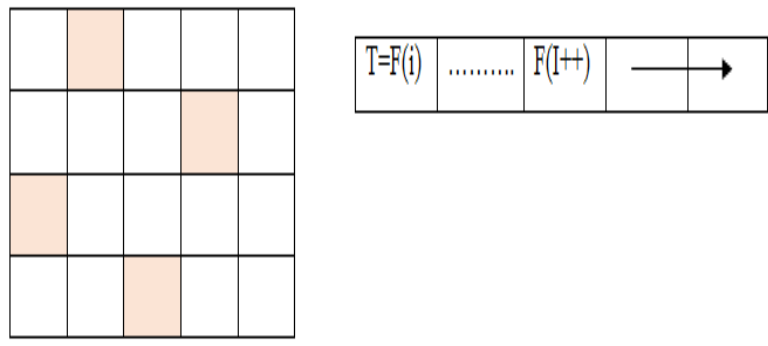

Figure 3 swapping terms using confidence measure

The swapping resembles the state of random projection which this swap states by maximum confidence of measure.

$$
\begin{array}{r}
T=i+ \pm \rightarrow \\
F(P \text { Optima } i, d t)<\mathrm{f}(G \text { Optima } i, d t) \ldots \ldots
\end{array}
$$

The most common type single point shortcut. Shortcut to a single point, mostly choosing swap state by frequent measures places that move the remaining sounds from one place to another. It's complicated and good for viewing.

Frequent words breakdown is based on the randomly chosen crossing point. This particular method is called a single point crossing and has only one cross-section. Sometimes the single child 1 or more is created, but often two generations are created, and new people are placed.Crossover is not always present, though. Sometimes, based on a set probability, no shortcut occurs, and parents are directly copied to new people. The probability of crossing is generally $60 \%$ to $70 \%$.

\subsection{Multiplicative Perturbations on frequent decision classifier}

The most common method of random point of perturbation makes the combination of adaptability between the state matrix represented storage multiplicative factors. But the privileges of privacy can use a good effect to safeguard privacy. The data arrangements of swapping factor resemble the matrix point of records order. The decision classifier decides the frequent terms to perturb the data to categorize records. The sharing of privacy of data mining can be greatly extended to privacy decisions making principle. 


\section{Algorithm}

Define $\mathrm{T} \rightarrow \max , \mathrm{X} \rightarrow \max$, and $\mathrm{X} \rightarrow$ min and objective function $\mathrm{f}$;

The data initialization multiplicative factor

For $\mathrm{i}=1$ to $\mathrm{N}$

$$
\begin{array}{r}
\text { Initialize } \mathrm{X}_{\mathrm{i}, \mathrm{d}} \text { and } \mathrm{V}_{\mathrm{i}, \mathrm{d}} \\
P_{\text {Optima } i, d}^{t}=\mathrm{Xi}, \mathrm{d}
\end{array}
$$

Evaluate fitness value (, )for all $\mathrm{N}$ elephant group positions;

Optima, $\mathrm{d}=\operatorname{Min}(\mathrm{x})$; Assign value of according to the weight update rules

For $=1$ to

Start the iteration

For $\mathrm{i}=1$ to $\mathrm{N}$ if rand $>\mathrm{p}$

Identify least points of data records

Else //Local Search optimal decision search or update the elephant velocity

end if; update the position, using Eq. (3);

Evaluate fitness value for (, );

Compute the max terms of redundant data terms $\mathrm{X} \rightarrow \mathrm{P}$

$$
\begin{aligned}
& \text { If }\left(X_{i, d}^{t}\right)<F\left(P_{\text {Optima } i, d}^{t}\right) \\
& F\left(P_{\text {Optima } i, d}^{t}\right)=X_{i, d}^{t}
\end{aligned}
$$

End if ;

Compute the $\mathrm{mx}$ terms of redundant data terms $\mathrm{P} \rightarrow \mathrm{G}$

$$
\begin{aligned}
& \text { If } F\left(P_{\text {Optima } i, d}^{t}\right)<\mathrm{f}\left(G_{\text {Optima } i, d}^{t}\right) \\
& G\left(P_{\text {Optima } i, d}^{t}\right)=P_{\text {Optima } i, d}^{t}
\end{aligned}
$$

End if

End for

$$
\mathrm{X}=G_{\text {Optima } i, d}^{t}
$$

Return $\mathrm{X}^{\mathrm{t}}$ and $F\left(P_{\text {Optima } i, d}^{t}\right)$

The above algorithm shows the evaluated points of least points frequent resemblance to note that random parameters are incorporated along with the redundant preserving data terms so that chance of sticking at local optima for the metaheuristics can be reduced by making the probability matrix.

\subsection{Crypto perturbation outsourced security}

The group-key-based random perturbation generation can address the first issue. The data providers share the same random seed (the group key) to generate the same perturbation locally. There is abundant literature on group key management so that will skip the details here. The perturbed data cannot be delivered to the service provider directly since the network is not secure and other data providers can log the transmitted data and recover the original data with the known perturbation. Thus, the perturbed data have to be encrypted with the public key provided by the service provider before it goes to the public.

The service provider decrypts the perturbed data with her private key and pools the data together to mine a unified model. The unified model is returned to the data providers. Since the unified model is in the perturbed space, before the data provider applies it to the new data, she needs to transform her new data with the unified perturbation. The mining procedure and the model application procedure will be the same for all protocols.

\section{RESULT AND DISCUSSION}

The test case results are observed through evaluation performed metrics with various conventional methods the experimental results are tested with differential dataset contains anonymous data collected from online UCI repository. The proposed methods tested with three types of datasets from frequent mining collected groups, the database is retail dataset (database 1), T10I4D100K (database 2), Chess (database 3), the implementation carried through visual studio framework with accord .net for casting SQL injector. The methods used the conventional methods for the comparison includes the Firefly Algorithm (FA), Particle Swarm Optimization (PSO), Elephant Herd Optimization, and Improved Apriori. The implementation represents the relative coordination that is measured with the comparative analysis that is progressed based on the performance metrics. The performance metrics are data integrity, false preservation rate, data hiding rate, time complexity, privacy standard accuracy. Privacy accuracy defines the overall performance from the integrity level part the false privacy state.

The performance measure is tested with average fixation of confidence value between the lower limitconfidence the upper limit confidence, the lower limit is $<50$ range $>100$. The proposed algorithm produces higher efficiency and performance compared to the other dissimilar methods. A formula gives the following equation can evaluate the Precision value.

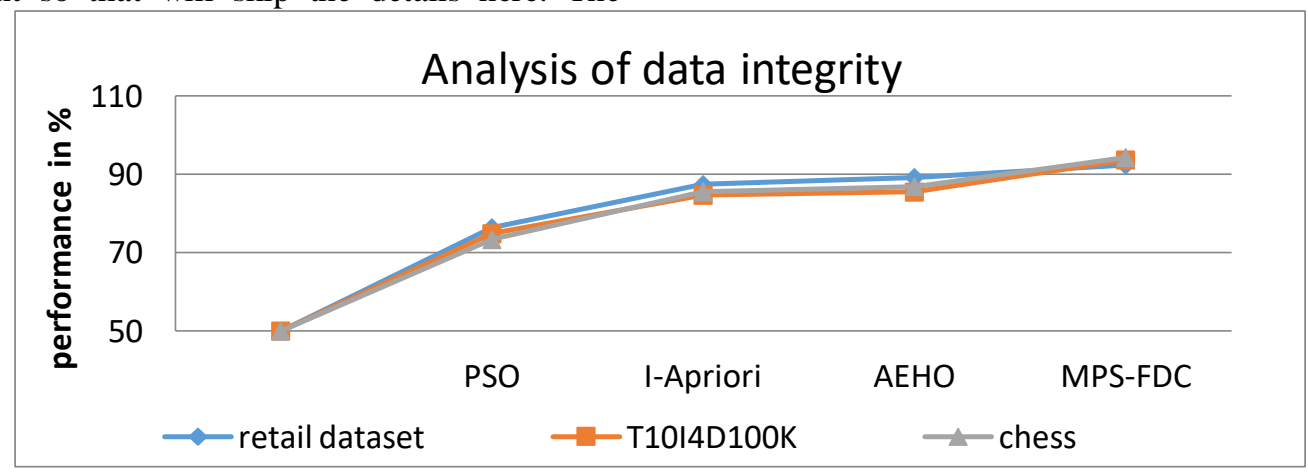

Figure 4: Evaluation of a data integrity 
Figure 4, represents the proposed method compared with other dissimilar ways. The performance of data integrityrate is higher resultant province than other methods has a significant impact. To find that most comments are in processed in repetitive generative comments are based on the transaction in the comments model

Table 5: comparison of data integrity

\begin{tabular}{|l|l|l|l|l|}
\hline & \multicolumn{4}{|c|}{ Impact of data integrity in \% } \\
\hline $\begin{array}{l}\text { Techniques } \\
\text { /datasets }\end{array}$ & PSO & $\begin{array}{l}\text { I- } \\
\text { Aprio } \\
\text { ri }\end{array}$ & $\begin{array}{l}\text { AEH } \\
\text { O }\end{array}$ & $\begin{array}{l}\text { MPS- } \\
\text { FDC }\end{array}$ \\
\hline Retail dataset & 76.3 & 87.3 & 89.1 & 92.3 \\
\hline T10I4D100K & 74.8 & 84.6 & 85.4 & 93.6 \\
\hline chess & 73.2 & 85.5 & 86.8 & 94.2 \\
\hline
\end{tabular}

system. The proposed system produce up to $94.2 \%$ well accuracy than other methods.

Table 5, evaluation of data integrity rate which this system produce higher efficiency compared to another

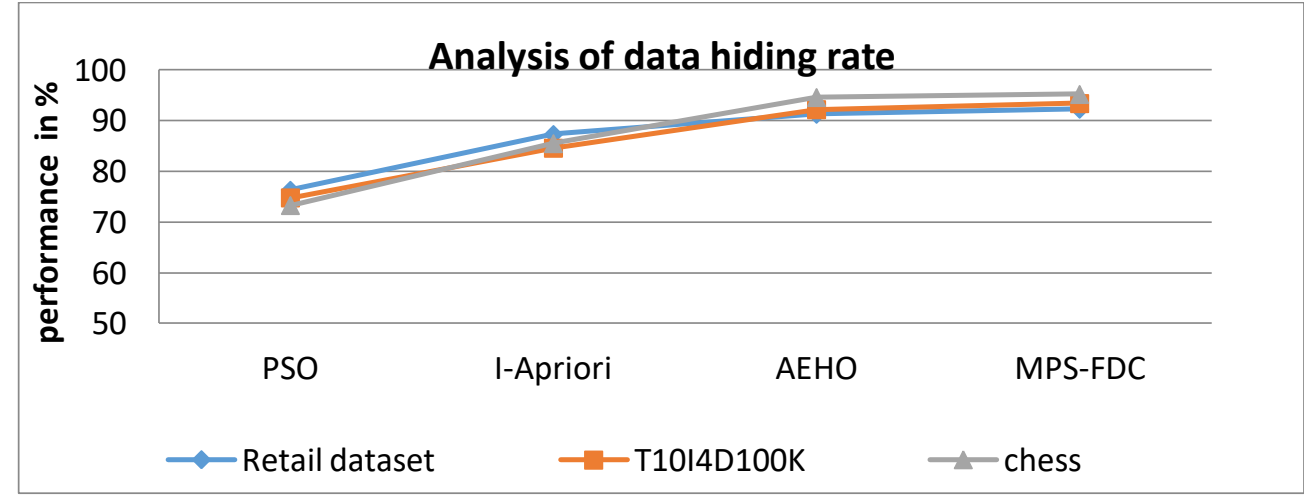

Figure 5: Comparison of data hiding rate

Figure 5, represents the proposed method compared with is higher resultant province than different ways has a significant impact. other dissimilar ways. The performance of data hiding rate

Table 6: comparison of data hiding rate

\begin{tabular}{|l|l|l|l|l|}
\hline & \multicolumn{4}{|c|}{ Impact of } \\
\hline $\begin{array}{l}\text { Techniques } \\
\text { datasets }\end{array}$ & PSO hiding ratein \% & $\begin{array}{l}\text { I- } \\
\text { Aprior } \\
\mathrm{i}\end{array}$ & AEHO & $\begin{array}{l}\text { MPS- } \\
\text { FDC }\end{array}$ \\
\hline Retail dataset & 76.3 & 87.3 & 91.3 & 92.3 \\
\hline T10I4D100K & 74.8 & 84.6 & 92.2 & 93.4 \\
\hline chess & 73.2 & 85.5 & 94.6 & 95.2 \\
\hline
\end{tabular}

produces higher recall state of evaluation up to 92.3

The above table 6 shows the comparison of data hiding rateanalyses by various techniques. The proposed system

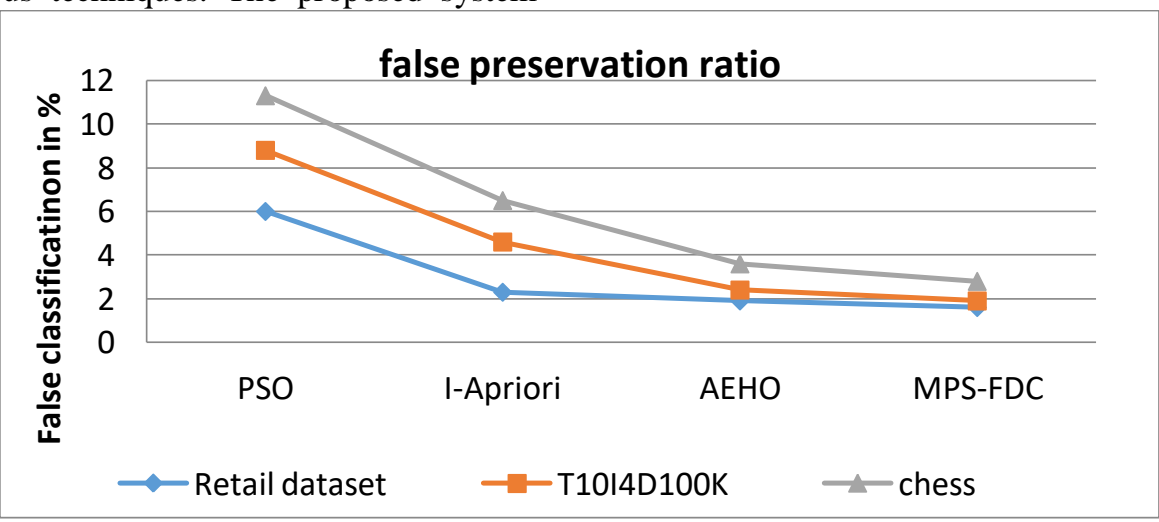

Figure 6: Comparison of false preservation 
Figure 6, represents the proposed method compared with other dissimilar ways. The performance of false extraction is lower resultant province than other methods has an enormous impact.

\begin{tabular}{|l|l|l|l|l|}
\hline & \multicolumn{3}{|c|}{$\begin{array}{l}\text { Comparison of false preservation } \\
\text { in } \%\end{array}$} \\
\hline $\begin{array}{l}\text { Techniques } \\
\text { /datasets }\end{array}$ & PSO & $\begin{array}{l}\text { I- } \\
\text { Apr } \\
\text { iori }\end{array}$ & $\begin{array}{l}\text { AEH } \\
\text { O }\end{array}$ & $\begin{array}{l}\text { MPS- } \\
\text { FDC }\end{array}$ \\
\hline Retail dataset & 6.6 & 5.3 & 5.2 & 4.4 \\
\hline T10I4D100K & 8.8 & 4.6 & 4.4 & 4.3 \\
\hline chess & 11.3 & 6.5 & 5.6 & 4.5 \\
\hline
\end{tabular}

Table 7: comparison of false preservation

Table 7, shows the comparison of the mean extraction ratio higher level of the state compared to the other methods .the evaluated performance shows that the proposed approach produces less false extraction ratio.

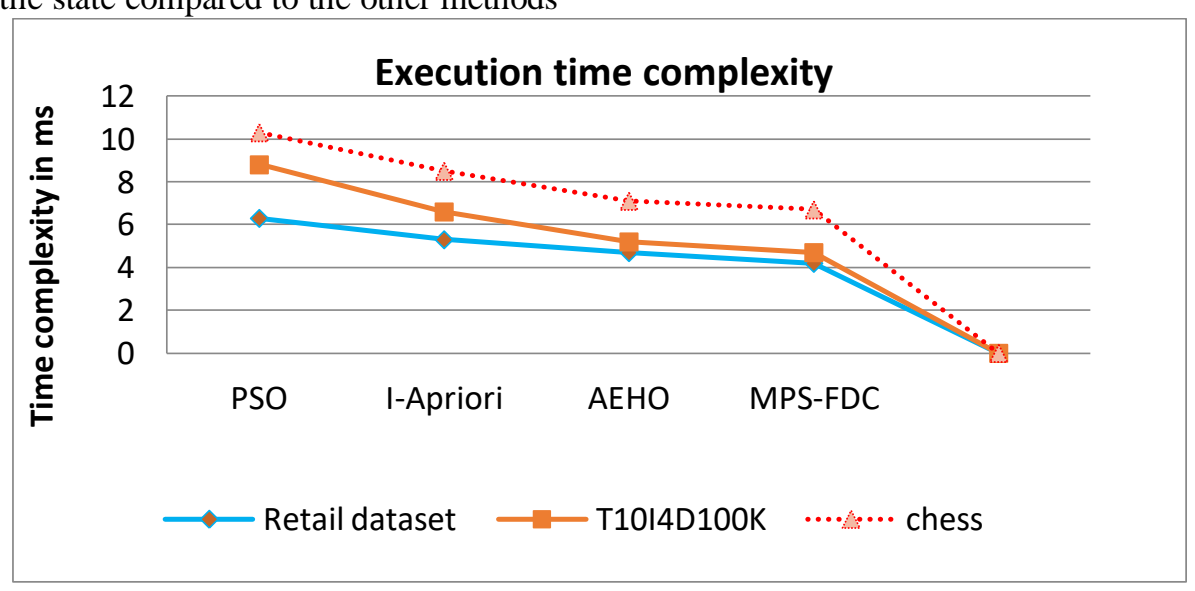

Figure 7: execution time complexity

complexity is lower resultant province than different ways has a great impact.

Figure 7, represents the proposed method is compared with other dissimilar ways. The performance of time

Table 8: execution of time complexity

\begin{tabular}{|c|c|c|c|c|}
\hline & \multicolumn{3}{|c|}{ Execution time complexity in seconds (ms) } \\
\hline Techniques /datasets & PSO & I-Apriori & AEHO & MPS-FDC \\
\hline Retail dataset & 6.3 & 5.3 & 4.7 & 4.2 \\
\hline T10I4D100K & 8.8 & 6.6 & 5.2 & 4.7 \\
\hline chess & 10.3 & 8.5 & 7.1 & 6.7 \\
\hline
\end{tabular}

Table 8 shows the time complexity of the dataset analyzed with different methods has a different preference of the proposed method. The implementation of the proposed method produces a higher performance with lower complexity.

\section{CONCLUSION}

The principal purpose of protecting the data tunnel is the algorithm to hide or provide privacy to some important information, which can not be exposed to unauthorized parties or infiltrators. Although the data mining case is a privacy and precision a couple of vague. Leads the mitigation occurs to others. In this case, a privacy protection attempt to make a privacy preserving in distributed grid data resource using multiplicative perturbation based on frequent decision classifier (MPS-FDC) have better effect thanof existing PPDM techniques. Finally, conclude that there is 
separate privacy that protects data usingefficient decision classification algorithms than all other methods, upto $95.7 \%$ efficiency, reliability, time complexity, and tolerance against privacy protocols than other algorithms on another specific privacy scale.

\section{REFERENCES}

1. H. Kargupta, S. Datta, Q. Wang, and K. Sivakumar, “on the privacy-preserving properties of random data perturbation techniques," in Proc. 3rd IEEE Int. Conf. Data Mining, Nov. 2003, pp. 99-106.

2. Stanley, R. M. O. and R. Z Osmar, "Towards Standardization in Privacy Preserving Data Mining," Published in Proceedings of 3rd Workshop on Data Mining Standards, WDMS' 2004, USA, p.7-17.

3. E. Bertino and I. N. Fovino, "Information driven evaluation of data hiding algorithms," in Proc. Int. Conf. Data Warehousing Knowl. Discovery, 2005, pp. 418427.

4. Helger Lipmaa," Cryptographic Techniques in PrivacyPreserving Data Mining," University College London, Estonian Tutorial 2007

5. C. C. Aggarwal and P. S. Yu, "A general survey of privacy-preserving data mining models and algorithms," in Privacy-Preserving Data Mining. New York, NY, USA: Springer, 2008, pp. 11-52.

6. B. C. M. Fung, K. Wang, R. Chen, and P. S. Yu, "Privacy-preserving data publishing: A survey of recent developments," ACM Comput. Surveys., vol. 42. no. 4, pp. 14:1-14:53, 2010.

7. Yaping L, Chen M, Li Q, Zhang W. Enabling Multilevel Trust in Privacy Preserving Data Mining. IEEE Transactions on Knowledge and Data Engineering. 2012, September; 24(9), $1598-1612$

8. Shikha Sharma \& Pooja Jain, "A Novel Data Mining Approach for Information Hiding," International Journal of Computers and Distributed Systems, Vol. No.1, Issue 3, October 2012

9. C.V.Nithya and A.Jeyasree, "Privacy-Preserving Using Direct and Indirect Discrimination Rule Method," Vivekanandha College of Technology for WomenNamakkal India, International Journal of Advanced Research in Computer Science and Software Engineering, vol.3 issue 12,2013. 